Discrete Comput Geom 30:185-193 (2003)

DOI: $10.1007 / \mathrm{s} 00454-003-0005-8$

\title{
Finite Packing and Covering by Congruent Convex Domains*
}

\author{
Károly Böröczky, Jr. \\ Rényi Institute of Mathematics, Hungarian Academy of Sciences, \\ Pf. 127, H-1364 Budapest, Hungary \\ carlos@renyi.hu
}

\begin{abstract}
For a convex domain $K$, let $H(K)$ be a circumscribed polygon with at most six sides whose area is minimal, and let $\tilde{H}(K)$ be an inscribed hexagon with at most six sides whose area is maximal. According to the celebrated result by L. Fejes Tóth [6], if a hexagon contains $n$ non-overlapping congruent copies of $K$, then its area is at least $n \cdot A(H(K))$, and if $n$ pairwise non-crossing congruent copies of $K$ cover a hexagon, then its area is at most $n \cdot A(\tilde{H}(K))$. Here two convex domains $C_{1}$ and $C_{2}$ are non-crossing if there exist complementary half-planes $l^{-}$and $l^{+}$such that $l^{-} \cap C_{1} \subset C_{2}$ and $l^{+} \cap C_{2} \subset C_{1}$. In this paper we generalize the results of $\mathrm{L}$. Fejes Tóth to packings inside or coverings of any convex domain provided that the number of copies is high enough. In the case of packings of centrally symmetric domains, our results are optimal. Finally, let $K$ be centrally symmetric, and let $D_{n}$ be the convex domain with minimal area containing $n$ non-overlapping congruent copies of $K$. Then we show that $R\left(D_{n}\right) / r\left(D_{n}\right)$ stays bounded as $n$ tends to infinity.
\end{abstract}

\section{Introduction}

For a given convex domain $K$, let $H(K)$ be a circumscribed polygon with at most six sides whose area is minimal. It is well known (see [6]) that if a polygon $P$ having at most six sides contains $n$ non-overlapping congruent copies of $K$, then

$$
A(P) \geq n \cdot A(H(K)) .
$$

In this paper we provide a stronger version of this theorem:

Theorem 1. If a convex domain D contains $n$ non-overlapping congruent copies of a convex domain $K$, then

$$
A(D) \geq n \cdot A(H(K))
$$

provided that $n \geq N$ where $N$ depends only on $K$.

* This research was supported by OTKA T 31984 and OTKA T 30012, Hungary. 
We show in Section 3 that the $N$ in Theorem 1 does depend on $K$ (even for centrally symmetric domains), and cannot be chosen to be an absolute constant. This fact was already known to L. Fejes Tóth.

The constant $A(H(K))$ is not optimal in Theorem 1 for the typical convex domain $K$ (in the sense of the Baire category, see [7] for typical properties of convex domains). This can be shown by extending the method of G. Fejes Tóth [4]. On the other hand, if $K$ is centrally symmetric, then one can choose $H(K)$ to be centrally symmetric according to Dowker [2]. Thus there exists a lattice tiling by translates of $H(K)$, and hence taking large domains as $D$ shows that the constant $A(H(K))$ is optimal in Theorem 1 in this case. The proof of Theorem 1 also yields

Corollary 2. For a centrally symmetric convex domain $K$ that is not a parallelogram, let $D_{n}$ be a convex domain with minimal area that contains $n$ non-overlapping congruent copies of $K$. Then

$$
c_{1} \cdot \sqrt{n}<r\left(D_{n}\right)<R\left(D_{n}\right)<c_{2} \cdot \sqrt{n},
$$

where the positive constants $c_{1}$ and $c_{2}$ depend on $K$.

Just as L. Fejes Tóth [6], we need some extra assumptions in case of coverings: We call two convex domains $C_{1}$ and $C_{2}$ non-crossing if there exist complementary half-planes $l^{-}$and $l^{+}$such that $l^{-} \cap C_{1} \subset C_{2}$ and $l^{+} \cap C_{2} \subset C_{1}$. We note that translates are always non-crossing. For a convex domain $K$, let $\tilde{H}(K)$ be an inscribed hexagon with at most six sides whose area is maximal.

Theorem 3. If a convex domain $D$ is covered by $n$ non-crossing congruent copies of a convex domain $K$, then

$$
A(D) \leq n \cdot A(\tilde{H}(K))
$$

provided that $n \geq \tilde{N}$ where $\tilde{N}$ depends only on $K$.

If $K$ is centrally symmetric, then one can choose $\tilde{H}(K)$ to be centrally symmetric according to Dowker [2]. Thus there exists a lattice tiling by translates of $\tilde{H}(K)$, and hence taking large domains as $D$ shows that the constant $A(\tilde{H}(K))$ is optimal in Theorem 3. On the other hand, the constant $A(\tilde{H}(K))$ is not optimal in Theorem 3 for the typical convex domain $K$. This can be shown by extending the method of G. Fejes Tóth and Zamfirescu [5].

The $\tilde{N}$ in Theorem 3 can be most probably chosen to be an absolute constant. For translative coverings, $\tilde{N}=26$ works according to G. Fejes Tóth [3].

It is widely believed that Theorem 3 holds without the assumption that the copies are non-crossing. This conjecture seems to be rather obvious to believe, yet it may happen that a convex domain $D$ can be covered by $n$ congruent copies of a convex domain $K$, but it cannot be done in a pairwise non-crossing manner. We recall the following example due to Heppes: Let $D$ be a unit square, and let $K$ be the convex hull of the midpoints of the sides of $D$ and two opposite vertices. Then $K$ and a rotated image by $\pi / 2$ cover $D$. On the other hand, it is not hard to see that two non-crossing copies of $K$ cannot cover $D$. 
The arguments in this paper are variations of the original proofs in [6]. In particular, they depend on the Dowker theorems (see [2]).

\section{Imitating a Cell Decomposition}

One of the fundamental observations in [6] was that the average side for a cell decomposition of a convex domain is at most six. First we improve on this bound if the number of the edges on the boundary are large.

A planar topological cell complex $\Sigma$ is a collection of finitely many two-cells, edges (the one-cells) and vertices (the zero-cells). The edges are Jordan arcs (that are convex arcs in this paper), and the endpoints of the edges are among the vertices. In addition, the two-cells are regions that are bounded by finitely many edges. The fundamental property that makes $\Sigma$ a cell complex is that the intersection of any two of the cells is a cell itself. We write $f_{i}(\Sigma)$ to denote the number of $i$-cells of $\Sigma$. Then the Euler formula yields

Proposition 4. Let $\Sigma$ be topological cell complex such that $\operatorname{supp} \Sigma$ is a convex domain, and each vertex is of degree at least three. We write $k_{1}, \ldots, k_{f_{2}(\Sigma)}$ to denote the numbers of sides of the two-cells, and $b$ to denote the number of edges of $\Sigma$ that are contained in exactly one two-cell. Then

$$
\sum_{i=1}^{f_{2}(\Sigma)}\left(6-k_{i}\right) \geq b+6
$$

Proof. Since $3 \cdot f_{0}(\Sigma) \leq 2 \cdot f_{1}(\Sigma)$, we deduce by the Euler formula $f_{0}(\Sigma)-f_{1}(\Sigma)+$ $f_{2}(\Sigma)=1$ that

$$
6 \cdot f_{2}(\Sigma) \geq 2 \cdot f_{1}(\Sigma)+6
$$

On the other hand, counting the number of sides of each two-cell shows that $\sum_{i=1}^{f_{2}(\Sigma)} k_{i}=$ $2 \cdot f_{1}(\Sigma)-b$.

The first step in order to prove Theorem 1 would be to define a cell decomposition of $D$ into convex cells such that each cell contains exactly one of the congruent copies of $K$. The only little problem is that such a cell decomposition may not exist, therefore we save the essential properties of a cell decomposition following the ideas in [6].

Lemma 5. Let $D$ be a convex domain that contains the non-overlapping convex domains $K_{1}, \ldots, K_{n}$. Then there exist non-overlapping convex domains $\Pi_{1}, \ldots, \Pi_{n} \subset D$ satisfying the following properties:

(i) $K_{i} \subset \Pi_{i}$

(ii) $\Pi_{1}, \ldots, \Pi_{n}$ cover $\partial D$.

(iii) $\Pi_{i}$ is bounded by $k_{i} \geq 2$ convex arcs that we call edges. The edges intersecting int $D$ are segments, and the rest of the edges are the maximal convex arcs of $\partial D \cap \Pi_{i}$. 
(iv) The number $b$ of edges contained in $\partial D$ satisfy

$$
\sum_{i=1}^{n}\left(6-k_{i}\right) \geq b+6 .
$$

Proof. Let $\Pi_{1}, \ldots, \Pi_{n}$ be non-overlapping convex domains such that $K_{i} \subset \Pi_{i} \subset D$, and the total area covered by the convex domains $\Pi_{1}, \ldots, \Pi_{n}$ is maximal under these conditions. Since two non-overlapping convex sets can be separated by a line, each $\Pi_{i}$ is the intersection of a polygon $P_{i}$ and $D$. Now int $P_{i} \cap \partial D$ consists of finitely many convex arcs whose closures we call edges of $\Pi_{i}$. The set of vertices of $\Pi_{i}$ consists of the endpoints of its edges in $\partial D$, and all the vertices of some $P_{j}$ that are contained in $\Pi_{i} \cap$ int $D$. These vertices divide $\partial \Pi_{i} \cap$ int $D$ into finitely many segments whose closures form the rest of the edges of $\Pi_{i}$.

Now $\Pi_{1}, \ldots, \Pi_{n}$ may not cover $D$, and the closure of a connected component of int $D \backslash \bigcup_{i=1}^{n} \Pi_{i}$ is called a hole. The maximality of $A\left(\Pi_{i}\right)$ yields that each hole $Q$ is a possibly non-convex domain that is bounded by finitely many convex arcs $s_{1}, \ldots, s_{k}$ with the following properties: $s_{j-1}$ and $s_{j}$ share a common endpoint for $j=1, \ldots, k$ (where $s_{0}=s_{k}$ ) that is a vertex of some $\Pi_{i}$, and no other intersection occurs among $s_{1}, \ldots, s_{k}$, and no other vertices of any $\Pi_{i}$ are contained in $Q$. We note that each $s_{j}$ is contained either in the boundary of $D$ or in a straight edge of $\Pi_{i}$.

We may assume that $s_{1}$ intersects int $D$, and is contained in the edge $e_{1}$ of $\Pi_{i_{1}}$. Since $A\left(P_{1}\right)$ is maximal, we deduce that one endpoint $v_{2}$ of $s_{1}$ lies in the relative interior of $e_{1}$. We may assume that $v_{2}$ is the common endpoint of $s_{1}$ and $s_{2}$. Then $s_{2}$ is contained in an edge $e_{2}$ of some $\Pi_{i_{2}}$ where $v_{2}$ is an endpoint of $e_{2}$, and the other endpoint $v_{3}$ of $s_{2}$ lies in the relative interior of $e_{2}$. Now we obtain by induction that $s_{j}$ is contained in an edge $e_{j}$ of some $\Pi_{i_{j}}$, and, for $j=2, \ldots, k$, the common endpoint $v_{j}$ of $s_{j-1}$ and $s_{j}$ is a vertex of $e_{j}$, and lies in the relative interior of $e_{j-1}$. We also deduce that the common endpoint $v_{1}$ of $s_{k}$ and $s_{1}$ is a vertex of $e_{1}$, and lies in the relative interior of $e_{k}$, and hence $Q$ is a convex polygon that is contained in the interior of $D$. In particular, we conclude that $\Pi_{1}, \ldots, \Pi_{n}$ cover $\partial D$.

Finally, in order to estimate the average number of sides of $\Pi_{1}, \ldots, \Pi_{n}$, we construct a related topological cell decomposition $\Sigma$ of $D$. If there exists no hole, then $\Pi_{1}, \ldots, \Pi_{n}$ defines a cell decomposition, and (iv) follows directly from Proposition 4. Otherwise let $\left\{Q_{1}, \ldots, Q_{m}\right\}$ be the set of holes, and let $q_{j} \in$ int $Q_{j}$. The idea for defining $\Sigma$ is to shrink each $Q_{j}$ to $q_{j}$. The two-cells of $\Sigma$ are $\Pi_{1}^{*}, \ldots, \Pi_{n}^{*}$, where $\Pi_{i}^{*}$ is the union of $\Pi_{i}$ and all triangles of the form $\operatorname{conv}\left\{q_{j}, s\right\}$ such that $s$ is a side of $Q_{j}$ and $e \subset \Pi_{i}$. We note that $\Pi_{i}^{*}$ and $\Pi_{j}^{*}$ do not overlap for $i \neq j$. Now an edge of $\Sigma$ is either an edge of some $\Pi_{i}$ contained in $\partial D$, or of the form $\Pi_{i}^{*} \cap \Pi_{j}^{*}$ for $i \neq j$ if it contains a segment.

Let us assume that the intersection of $\Pi_{i}^{*}$ and $\Pi_{j}^{*}, i \neq j$, contains a segment. Then $\Pi_{i}^{*} \cap \Pi_{j}^{*}$ is the union of $\Pi_{i} \cap \Pi_{j}$ and any segment $q_{k} v$ where the hole $Q_{k}$ has one-one sides in $\Pi_{i}$ and $\Pi_{j}$, and these two sides meet at $v$. We call the vertex $v$ of some $\Pi_{i}$ a dead vertex if it is the common vertex for two holes, and not the vertex for any other $\Pi_{j}$. Therefore the family of vertices for $\Sigma$ is the union of the vertices of $\Pi_{1}, \ldots, \Pi_{n}$ except for the dead vertices, and $\Sigma$ is actually a topological cell complex. Now $\Pi_{i}^{*}$ has at least as many edges as $\Pi_{i}$ has, and hence (iv) is a consequence of Proposition 4. 


\section{Packing Congruent Domains}

For a convex domain $K$ and $n \geq 3$, we write $t_{K}(n)$ to denote the minimal area of polygons with at most $n$ sides containing $K$. In particular, $t_{K}(6)=A(H(K))$. According to the Dowker theorem for circumscribed polygons (see [2]), $t_{K}(n)$ is a convex function of $n$. We define $t_{K}(2)=2 \cdot t_{K}(3)$. Since $t_{K}(3) \leq 2 \cdot t_{K}(4)$, the function $t_{K}(n)$ stays convex even for $n \geq 2$.

In order to prove Theorem 1, we may assume that $K$ is not a polygon with at most five sides, and hence $t_{K}(5)>t_{K}(6)$ holds. In this case we verify that there exist positive constants $\gamma_{1}$ and $\gamma_{2}$ depending on $K$ such that

$$
A(D)>n \cdot A(H(K))+\gamma_{1} \cdot P(D)-\gamma_{2} .
$$

We write $K_{1}, \ldots, K_{n}$ to denote the non-overlapping congruent copies of $K$, and we may assume that $D$ is the convex hull of these domains. Let $\Pi_{1}, \ldots, \Pi_{n}$ be the convex domains associated to $K_{1}, \ldots, K_{n}$ by Lemma 5 . In this proof, $\sigma$ always denotes an edge of some $\Pi_{i}$ that is contained in $\partial D$. Let $\sigma \subset \Pi_{i}$. We write $x_{i}$ to denote the centre of a circle with radius $r(K)$ inscribed into $K_{i}$, and $C\left(\sigma, x_{i}\right)$ to denote the union of all segments connecting $x_{i}$ to the points of $\sigma$. Now any tangent lines to $\partial D$ at the points of $\sigma$ avoid $K_{i}$, which in turn yields that

$$
A\left(C\left(\sigma, x_{i}\right)\right) \geq \frac{1}{2} \cdot r(K) \cdot|\sigma|
$$

where $|\cdot|$ stands for the arc length. We deduce that there exist positive constants $\lambda$ and $c_{1}$ such that if $|\sigma|>\lambda$, then

$$
A\left(C\left(\sigma, x_{i}\right)\right) \geq t_{K}(2)+c_{1} \cdot|\sigma| .
$$

Next we assume that $|\sigma| \leq \lambda$, and let $p$ and $q$ denote the endpoints of $\sigma$. The total curvature $\alpha(\sigma)$ is defined to be the variation of the angle of the tangent from $p$ to $q$ along $\sigma$ (it might be larger than $\pi$ ). If $\alpha(\sigma)>0$, then $\sigma$ intersects $\Pi_{i}$ because $D$ is the convex hull of $K_{1}, \ldots, K_{n}$. Therefore there exist positive $\alpha<\pi / 6$ and $\beta$ depending on $\lambda$ and $K$ satisfying the following property: if $\alpha(\sigma)<\alpha$, then the other edge of $\Pi_{i}$ at $p$ encloses an angle larger than $\beta$ with the segment $p q$, and a similar property holds for $q$. Let $Q(\sigma)$ be the quadrilateral that is bounded by the line $p q$, the lines of the other edges of $\Pi_{i}$ at $p$ and $q$, and the tangent of $\sigma$ that is parallel to $p q$. Then there exists a constant $c_{2}$ depending on $\lambda, \alpha$ and $\beta$ such that if $\alpha(\sigma)<\alpha$, then

$$
A(Q(\sigma)) \leq c_{2} \cdot \alpha(\sigma)
$$

We deduce by (3) and (4) that

$$
A\left(\Pi_{i}\right) \geq t_{K}\left(k_{i}\right)+\sum_{\substack{\sigma \subset \Pi_{i} \\|\sigma|>\lambda}} c_{1} \cdot|\sigma|-\sum_{\substack{\sigma \subset \Pi_{i} \\|\sigma| \leq \lambda \\ \alpha(\sigma)<\alpha}} c_{2} \cdot \alpha(\sigma)-\sum_{\substack{\sigma \subset \Pi_{i} \\|\sigma| \leq \lambda \\ \alpha(\sigma) \geq \alpha}} t_{K}(2) .
$$

Since the total curvature of $\partial D$ is $2 \pi$, it follows that

$$
A(D) \geq \sum_{i=1}^{n} t_{K}\left(k_{i}\right)+\sum_{|\sigma|>\lambda} c_{1} \cdot|\sigma|-c_{2} \cdot 2 \pi-\frac{2 \pi}{\alpha} \cdot t_{K}(2) .
$$


Now the concavity of $t_{K}(n)$ yields that

$$
t_{K}\left(k_{i}\right) \geq A(H(K))+\left(t_{K}(5)-t_{K}(6)\right) \cdot\left(6-k_{i}\right) .
$$

We write $b$ to denote the number of edges of $\Pi_{1}, \ldots, \Pi_{n}$ that are contained in $\partial D$. Then Lemma 5(iv) yields that

$$
\begin{aligned}
\sum_{i=1}^{n} t_{K}\left(k_{i}\right) & \geq n \cdot A(H(K))+\left(t_{K}(5)-t_{K}(6)\right) \cdot b \\
& \geq n \cdot A(H(K))+\sum_{|\sigma| \leq \lambda} \frac{t_{K}(5)-t_{K}(6)}{\lambda} \cdot|\sigma| .
\end{aligned}
$$

In turn, we conclude (2) by (5). Now $A(D) \geq n \cdot A(K)$ and the isoperimetric inequality yield that $P(D)>2 \sqrt{A(K) / \pi} \cdot \sqrt{n}$, therefore $A(D)>n \cdot A(H(K))$ holds for large $n$.

One may hope that the $N$ in Theorem 1 can be chosen to be an absolute constant. We now present an example showing that this is not the case, not even if $K$ is centrally symmetric: Let $\varepsilon>0$ be small, and consider in a coordinate system the convex hull of the points $p=(1,0), q=(0,1)$, and the hyperbole arc with the equation $x \cdot y=\varepsilon$ in the positive corner. We write $\gamma$ to denote the arc between $p$ and $q$ on the boundary. We define $K$ to be the centrally symmetric convex domain whose boundary consists of $\gamma$ and three other arcs congruent with $\gamma$. If $s$ is the segment with length $4(n-1)$ parallel to the first coordinate axis, then $s+K$ contains $n$ non-overlapping translates of $K$, and

$$
A(s+K)=(n-1) \cdot 4+A(K)=n \cdot 4-\varepsilon \cdot \ln \frac{1}{4 \varepsilon}-2 \varepsilon .
$$

On the other hand, any tangent to the hyperbole arc and the coordinate axes enclose a triangle of area $2 \varepsilon$, and hence $A(H(K)) \geq 4-12 \varepsilon$. Therefore the $N$ of Theorem 1 satisfies

$$
N \geq \frac{1}{12} \cdot \ln \frac{1}{4 \varepsilon}
$$

Finally, we investigate the shape of the optimal packing. Let $K$ be a centrally symmetric convex domain that is not a parallelogram, and let $D_{n}$ be a convex domain with minimal area that contains $n$ non-overlapping congruent copies of $K$.

We may assume that $H(K)$ is centrally symmetric according to Dowker [2], and hence there exits a tiling of the plane by translates of $H(K)$. If $B_{n}$ is a circle with minimal radius that contains $n$ tiles, then

$$
A\left(D_{n}\right) \leq A\left(B_{n}\right) \leq n \cdot A(H(K))+\gamma \cdot \sqrt{n},
$$

where $\gamma$ depends on $K$. We deduce by (2) that the perimeter of $D_{n}$ satisfies $P\left(D_{n}\right) \leq$ $\gamma^{\prime} \cdot \sqrt{n}$ for large $n$ and for some constant $\gamma^{\prime}$ depending on $K$. Therefore $r\left(D_{n}\right) \cdot P\left(D_{n}\right)>$ $A\left(D_{n}\right)$ yields that $r\left(D_{n}\right)>A(K) / \gamma^{\prime} \cdot \sqrt{n}$. We conclude $R\left(D_{n}\right)=O(\sqrt{n})$, which fact completes the proof of Corollary 2. 


\section{Coverings by Non-Crossing Congruent Domains}

For any finite non-crossing covering of a convex domain $D$, there exists an associated cell decomposition of $D$. This fact was observed by L. Fejes Tóth (see [6]) but his argument seemed to have some gaps. A rather long detailed proof was provided in [1]. Here we present an argument for the sake of completeness.

Lemma 6. Let $D$ be a convex domain that is covered by the pairwise non-crossing convex domains $K_{1}, \ldots, K_{n}$ such that no $(n-1)$ copies out of $K_{1}, \ldots, K_{n}$ cover $D$. Then there exists a cell decomposition of D into the two-cells $\Pi_{1}, \ldots, \Pi_{n} \subset D$, satisfying the following properties:

(i) $\Pi_{i} \subset K_{i}$.

(ii) We write $k_{i}$ to denote the number of edges of $\Pi_{i}$, and $b$ to denote the total number of edges contained in $\partial D$. Then

$$
\sum_{i=1}^{n}\left(6-k_{i}\right) \geq b+6
$$

Proof. There exists a covering $\Pi_{1}, \ldots, \Pi_{n}$ of $D$ by convex, compact sets such that each $\Pi_{i} \subset K_{i}$, the sets $\Pi_{1}, \ldots, \Pi_{n}$ are pairwise non-crossing, and $\sum_{i} A\left(\Pi_{i}\right)$ is minimal under the previous two conditions. Now each $\Pi_{i}$ is a convex domain because each $K_{i}$ is needed in order to cover $D$.

We suppose that there exist some $\Pi_{i}$ and $\Pi_{j}, i \neq j$, that overlap, and seek a contradiction. The idea is that we can cut off a small part of certain $\Pi_{i}$ in a way that the resulting family still forms a non-crossing cover of $D$. The difficulty is to ensure that the resulting family is pairwise non-crossing.

For $i<j$, we write $l_{i j}$ to denote some line that witnesses that $\Pi_{i}$ and $\Pi_{j}$ are noncrossing, and $l_{i j}^{+}$and $l_{i j}^{-}$to denote the half-planes such that $l_{i j}^{+} \cap \Pi_{j}$ contains $l_{i j}^{+} \cap \Pi_{i}$, and $l_{i j}^{-} \cap \Pi_{i}$ contains $l_{i j}^{-} \cap \Pi_{j}$, respectively. We may assume that $\Pi_{2}$ overlaps $l_{12}^{-}$, and hence there exists a supporting line $l$ to $\Pi_{2}$ that intersects $\Pi_{2}$ in a single point $p$ where $p \in \operatorname{int} l_{12}^{-}$.

After possibly renumbering the domains $\Pi_{3}, \ldots, \Pi_{n}$, let $\Pi_{2}, \ldots, \Pi_{m}, 2 \leq m \leq n$, form the family of $\Pi_{i}, i \geq 2$, such that $p \in \Pi_{i}$, and $p$ has a neighbourhood $U_{i}$ such that $\Pi_{i} \cap U_{i} \subset \Pi_{2} \cap U_{i}$. Then there exists a half-plane $\tilde{l}^{+}$such that the line $\tilde{l}$ bounding $\tilde{l}^{+}$is parallel to $l$, the interior of $\tilde{l}^{+}$contains $p$, and $\tilde{l}^{+} \cap \Pi_{i} \subset \tilde{l}^{+} \cap \Pi_{2}$ holds for $i=2, \ldots, m$. In addition we may assume that if a domain $\Pi_{k}$ or a line $l_{i j}$ does not contain $p$, then it does not intersect the cap $\tilde{l}^{+} \cap \Pi_{2}$.

We define $\Pi_{i}^{\prime}$ to be the closure of $\Pi_{i} \backslash \tilde{l}^{+}$if $i=2, \ldots, m$, and $\Pi_{i}^{\prime}=\Pi_{i}$ otherwise. Then $\Pi_{1}^{\prime}, \ldots, \Pi_{n}^{\prime}$ cover $D$ because $\tilde{l}^{+} \cap \Pi_{2}$ lies in $l_{12}^{-} \cap \Pi_{1}$. It also follows that each $\Pi_{i}^{\prime}$ is a convex domain and $\Pi_{i}^{\prime} \subset K_{i}$. Now $l_{i j}$ witnesses that $\Pi_{i}^{\prime}$ and $\Pi_{j}^{\prime}$ are non-crossing for any $i<j$ unless $2 \leq i \leq m, j>m, p \in l_{i j}$ and $\Pi_{j}^{\prime}=\Pi_{j}$ overlaps $l_{i j}^{-}$. In this case we distinguish two possibilities: If $\Pi_{i}^{\prime}$ and $\Pi_{j}^{\prime}$ do not overlap, then they are readily non-crossing. Otherwise, the half-line $\tilde{l} \cap l_{i j}^{-}$intersects $\partial \Pi_{j}$ in a point $p^{\prime} \in \operatorname{int} l_{i j}^{-}$. Let $q \neq p$ be the other endpoint of $l_{i j} \cap \Pi_{j}$, and hence the line $p^{\prime} q$ witnesses that $\Pi_{i}^{\prime}$ and $\Pi_{j}^{\prime}$ are non-crossing. Since the total area of $\Pi_{1}^{\prime}, \ldots, \Pi_{n}^{\prime}$ is less than the total area 
of $\Pi_{1}, \ldots, \Pi_{n}$, we have arrived at a contradiction. Therefore $\Pi_{1}, \ldots, \Pi_{n}$ determine a topological cell decomposition of $D$, and hence (ii) is a consequence of Proposition 4 .

For a convex domain $K$, we write $\tilde{t}_{K}(n)$ to denote the maximal area of an inscribed polygon with at most $n$ sides. In particular, $A(\tilde{H}(K))=\tilde{t}_{K}(6)$. We also define $\tilde{t}_{K}(2)=0$. Since $\tilde{t}_{K}(3) \geq \frac{1}{2} t_{K}(4)$, the function $\tilde{t}_{K}(n)$ is concave for $n \geq 2$ according to the Dowker theorem for inscribed polygons (see [2]).

In order to prove Theorem 3, we may assume that $D$ is not a polygon with at most five sides, and hence $\tilde{t}_{K}(6)>\tilde{t}_{K}(5)$. We may also assume that no $n-1$ out of the $n$ congruent copies cover $D$. Let $\Pi_{1}, \ldots, \Pi_{n}$ form the cell decomposition of Lemma 6 . We write $\Pi_{i}^{\prime}$ to denote the convex hull of the $k_{i}$ vertices of $\Pi$ where $\Pi_{i}^{\prime}$ might be a segment. Then $A\left(\Pi_{i}^{\prime}\right) \leq \tilde{t}_{K}\left(k_{i}\right)$ holds by definition, and hence the concavity of $\tilde{t}_{K}(n)$ yields that

$$
A\left(\Pi_{i}^{\prime}\right) \leq \tilde{t}_{K}(6)+\left(\tilde{t}_{K}(6)-\tilde{t}_{K}(5)\right) \cdot\left(k_{i}-6\right) .
$$

We conclude by Lemma 6(ii) that

$$
\sum_{i=1}^{n} A\left(\Pi_{i}^{\prime}\right) \leq n \cdot A(\tilde{H}(K))-\left(\tilde{t}_{K}(6)-\tilde{t}_{K}(5)\right) \cdot(b+6) .
$$

Let $\sigma$ be an edge of $\Sigma$ that is contained in $\partial D$, and write $\alpha(\sigma)$ to denote the total curvature of $\sigma$. Since $\sigma$ is contained in a translate of $K$, it is not hard to see that

$$
A(\operatorname{conv} \sigma) \leq c \cdot \alpha(\sigma)
$$

holds for some $c$ depending on $K$. Now the total curvature of $\partial D$ is $2 \pi$, and hence

$$
A(D) \leq \sum_{i=1}^{n} A\left(\Pi_{i}^{\prime}\right)+c \cdot 2 \pi
$$

Thus Theorem 3 follows by (7) if $b \geq c \cdot 2 \pi /\left(\tilde{t}_{K}(6)-\tilde{t}_{K}(5)\right)$. Finally let $b<c \cdot 2 \pi /$ $\left(\tilde{t}_{K}(6)-\tilde{t}_{K}(5)\right)$. Since the perimeter of $D$ is at most $b \cdot P(K)$, the isoperimetric inequality yields $A(D)<A_{0}$ where $A_{0}$ depends only on $K$. Therefore we may choose $\tilde{N}=$ $A_{0} / A(\tilde{H}(K))$.

\section{Acknowledgment}

I thank Attila Pór for correcting a mistake in a previous version.

\section{References}

1. R.P. Bambah and C.A. Rogers: Covering the plane with convex sets. J. London Math. Soc., 27:304-314, 1952.

2. C.H. Dowker: On minimum circumscribed polygons. Bull. Amer. Math. Soc., 50:120-122, 1944.

3. G. Fejes Tóth: Finite coverings by translates of centrally symmetric convex domains. Discrete Comput. Geom., 2:353-363, 1987. 
4. G. Fejes Tóth: Densest packings of typical convex sets are not lattice-like. Discrete Comp. Geom., 14:1-8, 1995.

5. G. Fejes Tóth and T. Zamfirescu: For most convex discs thinnest covering is not lattice-like. In: Intuitive Geometry, K. Böröczky and G. Fejes Tóth (eds), János Bolyai series, North-Holland, Amsterdam, 1994.

6. L. Fejes Tóth: Some packing and covering theorems. Acta Sci. Math. Szeged, 12:62-67, 1950.

7. P.M. Gruber: Baire categories in convexity. In: Handbook of Convex Geometry, P.M. Gruber and J.M. Wills (eds), North-Holland, Amsterdam, pp. 1327-1346, 1993.

Received August 18, 2000, and in revised form February 15, 2001, and March 21, 2001. Online publication July 10, 2003. 